die Aktivierung und dann die eigentliche Puparisierung auslöse, daß also Aktivierung und Puparisierung nur zwei verschiedene Wirkungen eines Hormons seien. Dann müßten allerdings unsere normalen, reaktionsbereiten Testlarven bereits Puparisierungshormon (= Aktivierungshormon) enthalten, und zwar weit mehr, als durch Injektion von $1 \mathrm{CE}$. zugeführt wird ${ }^{10}$; denn bei ruhender Epidermis (Tab. 3, Ia) ist mit 10 CE. nur 15\% Verpuppung zu beobachten! Es bleibt dann unverständlich, weshalb gerade die Abdomina kopfverpuppter Tiere so gut reproduzierbare Testergebnisse liefern; wenn sie bereits körpereigenes Hormon enthalten, sollte dessen Menge zufälligen Schwankungen unterliegen und damit auch die zur vollständigen Verpuppung benötigte, durch Injektion zuzuführende Dosis. Man kann aber feststellen, daß im Gegenteil die kopf-nichtverpuppten Tiere sehr viel ungleichmäßiger auf injiziertes Hormon ansprechen. Wir neigen deshalb zu der Ansicht, daß zwei verschiedene Wirkstoffe nacheinander auf die Epidermis einwirken.

Allerdings können wir E. B e cker nicht so weit folgen, daß wir die Aufgaben des Puparisierungshormons allein in der Pigmentierung und Verhärtung der Cuticula erblicken. Wie Dennell ${ }^{15}$ gezeigt hat, besteht dieser Prozeß in einer Gerbung der

15 R. Dennell, Proc. Roy. Soc. [London], Ser. B 134, 79 [1947].
Proteinlamellen der Cuticula, die mit einer Dunkelfärbung einhergeht. Die Gerbung wird durch Phenole oder Chinone bewirkt, die aus dem Tyrosinstoffwechsel stammen; sie läßt sich nachahmen durch Behandlung isolierter Hautstücke mit einer Lösung von Brenzcatechin, das als Substrat für die in der Haut vorhandene Tyrosinase und Polyphenol-Oxydase dient. Es war daher die Vermutung aufgetaucht, der in unseren Extrakten enthaltene Stoff sei gleichfalls nur ein Substrat für diese Fermentsysteme. Dagegen spricht zunächst die hohe Wirksamkeit unserer Präparate; $0,05 \gamma$ geben bereits deutliche Effekte. Die typischen Substrate, Brenzcatechin, Protocatechusäure und Tyrosin sind in unserem Test mit $100 \gamma$ ohne Wirkung. Die Beobachtung von Dennell, daß isolierte Hautstücke sich in Brenzcatechinlösung „puparisieren“, haben wir bestätigt; unsere Hormonlösung hat mit $400 \mathrm{CE}$./ $\mathrm{ccm}$ keine entsprechende Wirkung. Diese Unterschiede zeigen deutlich, daß der Angriffspunkt unserer Hormonpräparate ein ganz anderer ist als der der genannten Phenole.

Für ihre Hilfe bei der Bereitung und Auswertung der Extrakte danken wir Frl. In geborg $\mathrm{Brachmann}$. Die im Abschnitt II beschriebenen Versuche wurden gemeinsam mit Frl. stud. rer. nat. Ils e Dimigen, Leibniz-Kolleg der Universität Tübingen, im Rahmen einer Trimesterarbeit durchgeführt.

Herrn Prof. Butenand danken wir für das stete Interesse und die wohlwollende Förderung unserer Arbeit.

\title{
Beobachtungen über das Zink in den Langerhansschen Inseln des Pankreas und seine Beziehungen zur Inselfunktion
}

\author{
Von Helmut Maske \\ Aus der II. Medizinischen Klinik der Universität München \\ (Direktor: Prof. Dr. G. von Bergmann) \\ (Z. Naturforschg. 8 b, $96-104$ [1953]; eingegangen am 15. Januar 1953)
}

\author{
Meinem verehrten Lehrer, Herrn Professor Dr., Dr. h. c. Adolf Butenandt. \\ zum 50. Geburtstag
}

\footnotetext{
Verschiedene Beobachtungen bei Untersuchungen über den Dithizon- und Alloxan-Diabetes gaben Hinweise dafür, daß dem in den Langerhansschen Inseln des Pankreas histochemisch nachweisbaren Zink eine physiologische Bedeutung zukommt. Das Ziel der vorliegenden Arbeit war, weitere Hinweise für eine solche Rolle des Inselzinks zu gewinnen.

1. Es wurde an Kaninchen gefunden, daß das in den Granula vorliegende Inselzink morphologisch und funktionell auf das engste mit dem Insulin oder dessen unmittelbarer Vorstufe verknüpft ist.

2. Bei hungernden Tieren und nach funktionellen Belastungen in Form von intensiven protrahierten Glucosebelastungen verhielten sich die Insulingranula und das Inselzink gleich.

3. Die histochemisch mit Sicherheit beobachteten Differenzen zwischen dem Inselzink hungernder und mit Glucose belasteter Kaninchen waren durch quantitative Bestimmungen des
} 
Gesamtpankreaszinks nicht nachzuweisen; eine Erklärung dafür wurde mit der Tatsache gegeben, daß die Inseln nur etwa $2 \%$ des Gesamtpankreas ausmachen, so daß selbst stärkere Schwankungen nicht in Erscheinung treten müssen. Dagegen wird ein Anhalt für den tatsächlichen maximalen Zinkgehalt der Inseln gewonnen, der sich innerhalb der biologischen Schwankungsbreite des Gesamtpankreaszinks bewegen muß.

4. Aus der topographischen Verteilung in den Inselzellen und nach dem Verhalten des Inselzinks unter extremen physiologischen Bedingungen wird geschlossen, daß dem Zink jedenfalls beim Kaninchen eine Bedeutung bei der sogenannten basalen Insulinregulation zukommt.

5. Auf Grund bekannter Tatsachen über den Einfluß von Zink auf die physikalisch-chemischen Eigenschaften vom Insulin ist anzunehmen, daß das physiologische Insulindepot in den Inselzellen als Zinkkomplex vorliegt.

6. Da die Voraussetzung für eine Abgabe des Hormons an das Blut dessen Überführung in eine lösliche Form ist, wird eine primäre Änderung der Zinkbindung durch konkurrierende Komplexbildner postuliert.

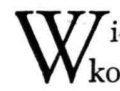
ie wir in verschiedenen früheren Arbeiten zeigen konnten, ist die Ausbildung des Dithizon- und Alloxan-Diabetes an die Gegenwart von Zink in den Langerhansschen Inseln gebunden.

Wenige Minuten nach der i.v. Injektion von Dithizon konnte der intensiv rot gefärbte Dithizon-ZinkKomplex in den Inseln nachgewiesen werden ${ }^{1}$. Der Nachweis für eine entsprechende Funktion des Inzelzinks bei der Entstehung des Alloxandiabetes wurde dadurch erbracht, daß nach einer vorhergehenden Eliminierung des Zinks aus den Langerhansschen Inseln kein Diabetes mit diabetogenen Alloxandosen mehr erzeugt werden konnte ${ }^{2}$.

Als Resultat dieser Untersuchungen ergeben sich die folgenden Überlegungen: 1. Substanzen mit spezifisch chemischer Affinität zum Zink werden in besonders zinkhaltigen Geweben z. B. in den Langerhansschen Inseln des Pankreas angereichert. Für diese Anreicherung sind wohl mehrere Faktoren von Bedeutung: das $p_{\mathrm{H}}$, spezifische physikalisch-chemische Eigenschaften der Zellen, die Gefäßversorgung u. a. 2. Um eine zellspezifische Wirkung zu erzielen, muß eine substanzspezifische Schwellenkonzentration erreicht werden.

Ähnliche schädigende Vorgänge wie an den Inseln werden nach der Injektion diabetogener Substanzen auch an anderen zinkreichen Geweben und Organen beobachtet; auch dort geht nach der Injektion von Dithizon die histochemisch nachweisbare Anreicherung der diabetogenen Substanz einer Schädigung voraus.

Eine Reihe von weiteren Beobachtungen, die wir während dieser Untersuchungen machen konnten, deuteten darauf hin, daß dem sehr hohen Zinkgehalt

1 B. St a mpfl, Ber. 36. Tagung Dtsch. Ges. Pathol. 1952.

2 H. M a s e, H. W o lff u. B. S t a m p f l, Klin. Wschr. 31, 79 [1953].

3 D. A. S cott u. A. M. F is her, Biochem. J. 29, 1048 [1935]. der Langerhansschen Inseln auch eine physiologische Bedeutung zukommt. Mit Hilfe einer neu ausgearbeiteten nativen Anfärbungsmethode konnte die Verteilung des Zinks innerhalb der Zellen genau untersucht werden. Das Zinkdithizonat liegt dabei in feingranulärer Form im Zellprotoplasma. Es besteht somit Veranlassung anzunehmen, daß dieselben Zellgranula, die als das spezifische Produkt dieser inkretorischen Drüsenzellen gelten, auch Träger des Zinks sind, und daß sich das Metall außerhalb der Granula in den Zellen jedenfalls nicht nachweisen läßt. Durch die Beobachtung einer so konzentrierten Lokalisation des Zinks und einer so engen topographischen Beziehung zwischen Insulin und Zink gewannen ältere Arbeiten, die sich bereits mit Insulin und Zink befaßt hatten, bei denen aber derartige Methoden nicht angewandt wurden, erneut an Interesse.

$\mathrm{S}$ c o $t \mathrm{t}$ und $\mathrm{F}$ is h e r beschrieben die Wirkung von geringen Zinkmengen auf die Kristallisation von Insulin ${ }^{3}$. Zink läßt sich dabei durch andere Metalle ersetzen, die aber auf Grund der im Pankreas gefundenen Mengen anscheinend keine physiologischen Beziehungen zum Insulin haben. Dieselben Verff. fanden im Pankreas von Diabetikern weniger Zink als bei normalen Kontrollen ${ }^{4}$. Diese letztere Beobachtung wurde bei der Nachuntersuchung durch Eisen brand, S i enz und We gel nicht bestätigt, da sie einer statistischen Betrachtung an einem größeren Material nicht standhielt ${ }^{5}$. E is e n bra nd und Mitarbeiter untersuchten und diskutierten die verschiedenen Möglichkeiten der Zinkbindung an Insulin und kamen zu dem Schluß, daß das Zink im Pankreas immer im Übergewicht gegenüber dem vorhandenen Insulin stehen muß6. Über die Bedeutung des Zinks, das auch in amorphen Insulinen und solchen, die mit der Brucinmethode kristallisiert wurden, vorkommt, ist bis heute nichts bekannt. Die blutzuckersenkende Wirkung des Insulins ist jedenfalls nicht von dessen Zinkgehalt ab-

4 D. A. S c o t t u. A. M. F i s h e r, J. clin. Invest. 17, 725 [1938].

5 J. Eisenbrand, M. Sienz u. F. Wegel, Med. u. Chem. 4, 259 [1942].

6 J. E i s e n bra nd u. F. W e g e 1, Hoppe-Seyler's Z. physiol. Chem. 268, 26 [1941]. 
hängig, wenn man von einem retardierenden Effekt absieht. Neuerdings wurde von W e it z e l auf Grund eigener experimenteller Arbeiten der Zinkgehalt des Insulins mit dessen initialer hyperglykämischer Wirkung in Verbindung gebracht 7 .

Für unsere Problemstellung sind besonders jüngste Untersuchungen von $\mathrm{H}$ a $\mathrm{ll}$ a s - M öll e r interessant, der zeigen konnte, daß ein erhöhter Zinkgehalt des Insulins (2 mg/1000 E) auch ohne Zusatz von anderen Depotkörpern bis über ein $p_{\mathrm{H}}$ von 8,0 hinaus das Insulin fällt. Voraussetzung dafür ist allerdings die Abwesenheit von Phosphat- und Citrationen, die anscheinend die Zinkbindung des Insulins verändern ${ }^{8}$. Diese Beobachtung zeigt, daß Insulin mit einem Überschuß von Zink bei physiologischem $p_{\mathrm{H}}$ unlöslich ist. Cohn wies kürzlich auch auf die Bedeutung der Tatsache hin, daß Zink bei neutralem $p_{\mathrm{H}}$ mit Proteinen schlecht lösliche Komplexe bildet 9 .

Im weiteren Verlauf unserer Untersuchungen fanden wir, daß Zink- und Insulingehalt der Inselzellen ein weitgehend paralleles Verhalten aufweisen. Wir konnten eine ältere Beobachtung, nach der das Inselzink nahrungsabhängigen Schwankungen unterworfen ist, bestätigen ${ }^{10}$ und darüber hinaus durch protrahierte Belastungen mit Glucose, die nach früheren Untersuchungen anscheinend das körpereigene spezifische Substrat für die Abgabe von Insulin an das Blut ist, das Zink gemeinsam mit dem Insulin weitgehend aus den Inseln eliminieren.

\section{Methoden und Material}

Die Untersuchungen wurden an 1,5-3,0 kg schweren Kaninchen beiderlei Geschlechts durchgeführt. Nach Möglichkeit wurden etwa $2,0 \mathrm{~kg}$ schwere Tiere verwendet. Auf Grund früherer Erfahrungen wurden mischrassige, bunte Kaninchen zu den Versuchen herangezogen.

Die kombinierten Glucosebelastungen wurden in der folgenden Weise durchgeführt: $24 \mathrm{Stdn}$. nüchterne Kaninchen erhielten 2-stdl. je $20 \mathrm{ccm} 20$-proz. Glucose langsam i.v. in eine Ohrrandvene und $20 \mathrm{ccm} 30$-proz. Glucose mit einem Tiemann-Katheter als Schlundsonde in den Magen. Wenn die Tiere wesentlich schwerer oder leichter als 2,0 kg waren, wurde die Menge der verabreichten Glucoselösungen dementsprechend erhöht oder herabgesetzt. Diese doppelten Glucosegaben wurden insgesamt 6-mal durchgeführt, so daß die Gesamtbelastung $10 \mathrm{Stdn}$. dauerte. Bei der letzten Belastung wurde Glucose lediglich i.v. gegeben, da bis zu dem Zeitpunkt der Tötung keine wesentliche Resorption vom Darm aus erwartet werden konnte. Im allgemeinen wurden Blutzucker genau im zeitlichen Mittelpunkt zwischen jeweils 2 Belastungen bestimmt.

7 G. W e it z e l, persönliche Mitteilung, Hoppe-Seyler's Z. physiol. Chem., im Druck.

$8 \mathrm{~K}$. Hallas-Möller, K. P etersen u. J.S chlichtkrull, Ugeskr. Laeger 113, 1761 [1951].

9 E. J. Cohn, K. S c h mid, W. H. B a t c h e l o r u. E. H. A l a me ry, Res. Comm. II. Congr. Int. Biochem. 1952.
Die Tiere wurden $1 / 2$ bis 1 Stde. nach der letzten Glucosegabe durch Luftembolie getötet und sofort seziert. Von dem sorgfältig soweit als möglich vom Fett freipräparierten Pankreasschwanz wurden jeweils 2 Stückchen für eine quantitative Zinkbestimmung, 1 Probe für eine Stickstoffbestimmung und der Rest für die histochemische Untersuchung verwendet.

Die quantitativen Zinkbestimmungen wurden mit der von Wolff angegebenen Methode durchgeführt11,12. Der Stickstoff wurde mit Hilfe des Mikrokjeldahlverfahrens, der Blutzucker nach $\mathrm{H}$ a gedorn-J en s en bestimmt.

Die histochemische Darstellung des Inselzinks erfolgte nach dem etwas abgeänderten Verfahren von $\mathrm{Ok}$ moto ${ }^{13}$. Um eine annähernd quantitative Beurteilung des histochemisch nachweisbaren Inselzinks durchführen zu können, wurden nur Schnitte gleicher Dicke (etwa $6 \mu$ ) herangezogen, und außerdem immer ältere Präparate als vergleichbarer Standard mitgefärbt.

Genauere Untersuchungen über die Verteilung des Zinks innerhalb der Inselzellen wurden an nativ gefärbten Tieren durchgeführt. Zu diesem Zweck wurde den lebenden Tieren eine entsprechende Dithizondosis injiziert und die Tiere etwa 10 Min. danach getötet. Über diese Methode und weitere damit erzielte Ergebnisse wird Stampfl an anderer Stelle berichten.

Bei der statistischen Auswertung der Ergebnisse wurde so vorgegangen, daß die bei einer Einteilung in Klassen völlig aus dem Rahmen fallenden Ergebnisse eliminiert wurden. In jeder Gruppe war es lediglich ein Tier, von dem jeweils beide Zinkwerte herausfielen. Im übrigen wurden die von verschiedenen Gewebsstückchen derselben Tiere gewonnenen Doppelwerte in der Statistik selbständig behandelt; dieses Vorgehen erscheint berechtigt, da nach den histochemischen Untersuchungen die Inseln ein und desselben Pankreas kein durchgehend gleiches Verhalten zeigten, eine Beobachtung, die weiter unten diskutiert und die mit der alternierenden Funktion der Inseln erklärt wird.

Die mittlere Abweichung vom Mittelwert $(=3 \sigma)$ wurde nach der folgenden Formel berechnet.

$$
\sigma_{x}= \pm \sqrt{\frac{1}{n} \sum_{i=1}^{n}\left(M-x_{i}\right)^{2}}
$$

$$
\text { Ergebnis se }
$$

\section{A) $\mathrm{H}$ istochemische Beobachtungen}

Bei der histochemischen Darstellung des Inselzinks mit der von $\mathrm{O} k$ a moto angegebenen Methode findet man selbst bei stärkeren Vergrößerungen eine mehr oder minder diffuse Verteilung des roten ZinkDiphenylthiocarbazid-Komplexes innerhalb der Zel-

$10 \mathrm{~K}$. O k a moto, Trans. Jap. Pathol. Soc. 33, 247 [1943].

11 H. W olff, Biochem. Z. 320, 291 [1950].

12 H. W olff u. G. B us e, Biochem. Z. 322, 154 [1951].

$13 \mathrm{~K}$. O k a moto, Trans. Jap. Pathol. Soc. 32, 99 [1942]. 
len, eine Tatsache, die wohl mit der Einwirkung der Fixation auf die feinere Zellstruktur in Zusammenhang gebracht werden muß; gegenüber dem exokrinen Gewebe sind die Inseln allerdings auch mit dieser Methode scharf abgegrenzt (Abb. ${ }^{*}$ ). Das letztere erscheint nur dann ganz leicht und diffus gerötet, wenn zwischen dem Tod des Tieres und der Fixation mehrere Stunden verstrichen sind. Unter dem Einfluß der Autolyse findet dann anscheinend eine Diffusion des Zinks aus den Inseln in das exokrine Gewebe statt.

Bei der Betrachtung nativ gefärbter Gefrierschnitte des Pankreas erhält man sehr viel schönere Ergebnisse. Da die Feinstruktur bei nativen Gefrierschnitten bekanntlich besser erhalten ist als bei fixiertem Material, läßt sich mit Hilfe dieser Methode die Verteilung des Zinks innerhalb der einzelnen Zellen studieren. Man findet, daß die Inselzellgranula, die nach früheren Untersuchungen das Inkret, also das Insulin oder eine Vorstufe desselben verkörpern, Träger des roten Farbstoffes sind, der spektralphotometrisch und emissionsspektrographisch nach seiner Extraktion mit Tetrachlorkohlenstoff als Zinkdithizonat charakterisiert werden konnte, wie $\mathrm{Sta} \mathrm{mpfl}$ in einer methodischen Arbeit ausführlich berichten wird (Abb. 2 und 3). Außerhalb der Granula war histochemisch beim Kaninchen kein Zink nachzuweisen. Wir konnten gleichzeitig durch die ausschließliche Darstellung der Granula deren Verteilung besonders gut studieren und fanden ältere Beobachtungen bestätigt, nach denen die Granula in der Hauptsache in der Nähe von Kapillaren lokalisiert sind; es fanden sich auch rotgefärbte Granula, in deren Nähe keine Kapillaren waren, aber auch diese lagen immerhin an der Peripherie der Zellen.

Normale und gut gefütterte Kaninchen zeigten reichlich und intensiv gefärbte Granula in ihren Inseln (Tab. 1). Die Inseln waren aber nicht gleichmäßig stark gefärbt, ein Befund, der bei dieser Gruppe von Tieren besonders auffiel, und der sicher nicht mit einer zufälligen Verteilung des Farbstoffes zusammenhängt, da er mit beiden Methoden, der nativen Anfärbung und der Okamoto-Methode, gleichermaßen zu erheben war. Wir möchten diese Beobachtung viel eher mit einer alternierenden Funktion verschiedener Inseln desselben Pankreas in $\mathrm{Zu}$ sammenhang bringen. Eine solche Annahme läßt sich anatomisch mit der Beobachtung von Ferner begründen, der am Abgang der Läppchenarterien von

* Abb. $1-3$, s. Tafel S. 100 a u. b.

14 H. F e rn e r, Virchow's Arch. pathol. Anatom. Physiol. klin. Med. 309, 87 [1942]. den interlobulären Gefäßen bei Mensch und Säugern häufig kleine Intimapolster von zirkulären glatten Muskelzellen, sogenannte Sperrwülste, fand ${ }^{14}$, die für eine abwechselnde Funktion verschiedener Inseln ursächlich verantwortlich gemacht werden können.

Die Langerhansschen Inseln 24-48 Stdn. hungernder Kaninchen enthalten vielleicht noch mehr Zink als die gefütterter Tiere (Tab. 2). Die Inseln erscheinen auch gleichmäßiger gefärbt als bei der vorhergehenden Gruppe. Freilich ist quantitativen Wertungen gegenüber am histologischen Schnitt, sofern es sich nicht um ganz grobe Unterschiede handelt, Vorsicht am Platze.

Sehr viel deutlicher ist der Unterschied zwischen den beiden ersten Gruppen und den Tieren, die in der angegebenen Weise über $10 \mathrm{Stdn}$. gleichzeitig peroral und i.v. mit Glucose belastet wurden. In einzelnen Inseln dieser Kaninchen war überhaupt kein Zink mehr nachzuweisen. Auch die Zellgranula waren in ihrer Zahl reduziert. Andere Inseln freilich zeigten nur eine geringe Veränderung des Zinkgehaltes. Bei aller Vorsicht in der quantitativen Bewertung histologischer Präparate war doch eine Differenz zwischen dem Zinkgehalt und der Zahl der Granula bei mit Glucose belasteten und bei unvorbehandelten Kaninchen deutlich nachzuweisen (Tab. 3).

Es sei an dieser Stelle noch einmal auf das bereits früher von uns beobachtete Verhalten des Inselzinks im Verlauf des Dithizon- und Alloxan-Diabetes hingewiesen. 6-12 Stdn. nach der Injektion der diabetogenen Substanzen kommt es in der Regel zur Ausbildung einer oft tödlichen Hypoglykämie bei den Versuchstieren. $\mathrm{Zu}$ dieser Zeit findet man auch eine sicher nachweisbare Abnahme des histochemisch nachweisbaren Inselzinks. Da die Hypoglykämie im Verlauf der Entstehung des experimentellen Diabetes heute hauptsächlich auf eine Abgabe des Insulins aus den zugrunde gehenden Inseln zurückgeführt wird, läßt sich auch hier wieder ein paralleles Verhalten von Insulin und Inselzink beobachten. Bei Tieren, die bereits $1-2$ Tage diabetisch sind, die also histologisch bei einer entsprechenden Dosierung der diabetogenen Substanzen nur mehr B-Zellenreste aufweisen, findet man kaum noch histochemisch nachweisbares Zink in den Langerhansschen Inseln ${ }^{15,16}$.

$15 \mathrm{H}$. Wolff, H. Maske, B. St a mpfl u. F. B a umg a rten, Naunyn-Schmiedebergs Arch. exp. Pathol. Pharmakol. 216, 440 [1952].

16 H. Maske, H. Wolff, B. Stampfl u. F. Baumg a rten, Naunyn-Schmiedebergs Arch. exp. Pathol. Pharmakol. 216, 457 [1952]. 


\begin{tabular}{|c|c|c|c|c|c|}
\hline $\begin{array}{l}\text { Tier } \\
\text { Nr. }\end{array}$ & $\begin{array}{c}\text { Gewicht } \\
\mathrm{kg}\end{array}$ & $\begin{array}{r}\mathrm{Zn} \\
\gamma / \mathrm{g}\end{array}$ & $\begin{array}{c}\mathrm{N}_{2} \\
\mathrm{mg} / \mathrm{g}\end{array}$ & Histochemischer Befund & Bemerkung \\
\hline 305 & 1,65 & $\begin{array}{l}62,4 \\
49,7\end{array}$ & 33,5 & - & \multirow{10}{*}{$\begin{array}{l}\text { fettiges Pankreas } \\
\text { wenig Fett }\end{array}$} \\
\hline 306 & 1,82 & $\begin{array}{l}34,7 \\
40,7\end{array}$ & 33,8 & + & \\
\hline 307 & 1,91 & $\begin{array}{l}34,3 \\
37,7\end{array}$ & 31,0 & ++-+++ & \\
\hline 308 & 2,28 & $\begin{array}{l}30,3 \\
37,8\end{array}$ & 34,2 & ++-+++ & \\
\hline 309 & 1,83 & $\begin{array}{l}21,9 \\
14,0\end{array}$ & 28,1 & +-++ & \\
\hline 320 & 1,85 & $\begin{array}{l}51,4 \\
55,3\end{array}$ & 37,5 & +++-+++ & \\
\hline 321 & 1,97 & $\begin{array}{l}61,7 \\
43,3\end{array}$ & 31,6 & ++-+++ & \\
\hline 322 & 2,18 & $\begin{array}{l}44,8 \\
45,3\end{array}$ & - & ++-+++ & \\
\hline 323 & 1,87 & $\begin{array}{l}45,7 \\
55,3\end{array}$ & 25,8 & +++-++++ & \\
\hline 324 & 1,92 & $\begin{array}{c}46,9 \\
42,9 \\
m=45,6 \\
\pm 24,3 \gamma / g\end{array}$ & $\begin{array}{c}28,5 \\
m=31,7 \\
\pm 9,9 \mathrm{mg} / \mathrm{g}\end{array}$ & +++ & \\
\hline
\end{tabular}

Tab. 1. Normale, gefütterte Kaninchen.

Eine sichere Differenzierung der zinkhaltigen Inselzellen in A- und B-Zellen steht allerdings noch aus. Dieses Problem hat sich experimentell als schwer lösbar gezeigt.

B) Quantitative Untersuchungen von Zink und Stickstoff im Kaninchenpankreas

Die quantitativen Untersuchungen konnten naturgemäß nur an Teilen des Gesamtpankreas durchgeführt werden. Die Inseln machen nur etwa $2 \%$ der Bauchspeicheldrüse aus ${ }^{17}$. Es war deshalb von vornherein fraglich, ob selbst starke Änderungen im Zinkgehalt dieser $2 \%$ bei quantitativen Bestimmungen an der Gesamtdrüse zum Ausdruck kommen, obwohl im exkretorischen Gewebe mit unseren Methoden histochemisch kein Zink zu finden ist. Tatsächlich ergaben sich keine signifikanten Unterschiede (Tab. 1-3, Abb. 4).

Ein Vergleich der histochemischen mit den quantitativen Befunden mußte aber jedenfalls einen Anhalt dafür geben, wieviel Zink die Inseln maximal

17 H. F er ner, Das Inselsystem d. Pankreas. G. Thieme 1952.

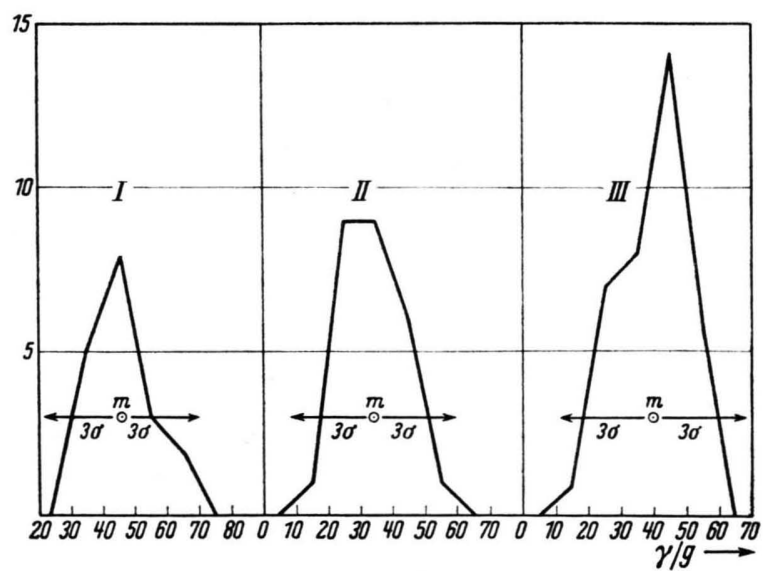

Abb. 4. Aufteilung der quantitativ bestimmten Zinkwerte im Gesamtpankreas nach Klassen von jeweils $1-10 \gamma / \mathrm{g}$. Die arithmetischen Mittel der Gruppen $(=m)$ und deren mittlere Abweichungen $(=3 \sigma)$ wurden jeweils eingetragen. I. normale, gefütterte Tiere, II. hungernde Tiere, III. mit Glucose belastete Tiere.

enthalten. Das heißt, wenn histochemisch nachweisbare extreme Schwankungen in dem Inselzink verschiedener Versuchsgruppen bei der quantitativen Zinkbestimmung im Gesamtpankreas nicht in Erscheinung treten, kann das Inselzink höchstens im Bereich 
H. Maske, Beobachtungen über das Zink in den Langerhansschen Inseln des Pankreas und seine Beziehungen zur Inselfunktion (S. 96)

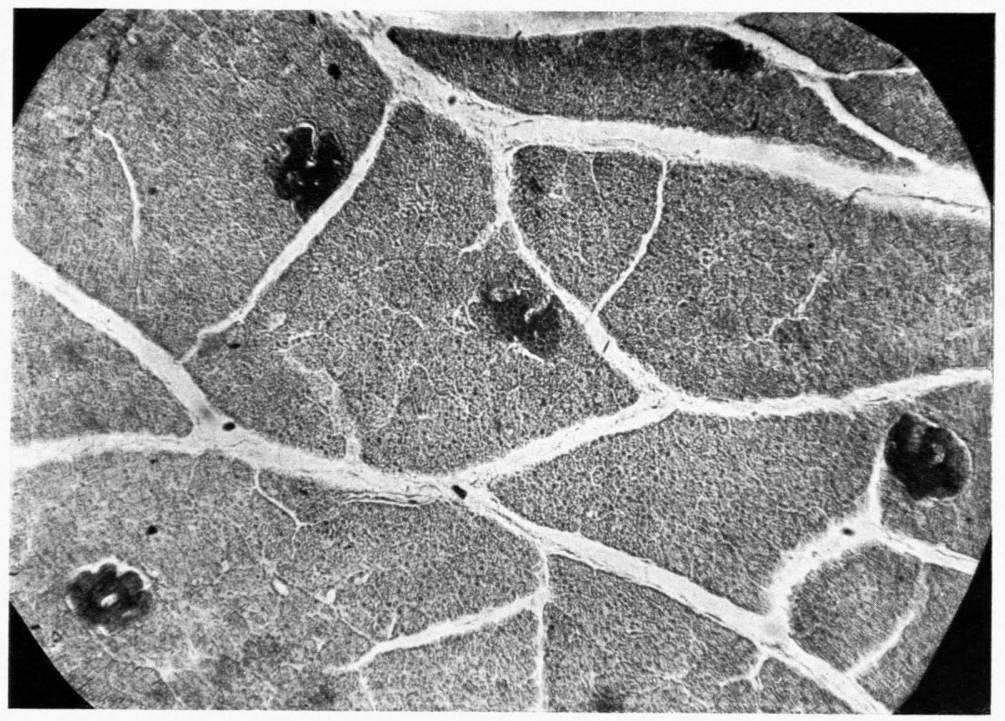

Abb. 1. Pankreas von Normaltieren, nach Oka moto auf Zink gefärbt; Vergrößerung $63 \times$, Grünfilter. Die Inseln heben sich scharf vom exkretorischen Parenchym ab.

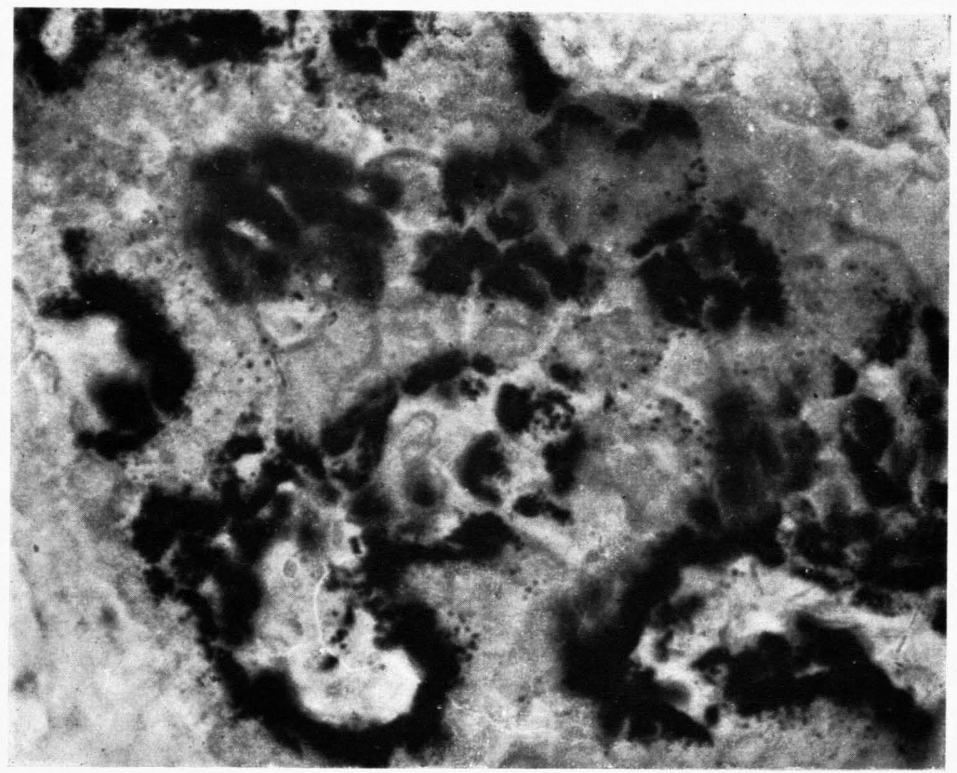

Abb. 2. Pankreas vom nativ gefärbten Normaltier, Inselausschnitt, Gefrierschnitt, Schnittdicke $25 \mu$, Vergrößerung $720 \times$, Immersion, Grünfilter. Die schwarzen Säume bestehen aus einzelnen Granulis, durch die dichte Lagerung derselben entsteht der kompakte Eindruck. 


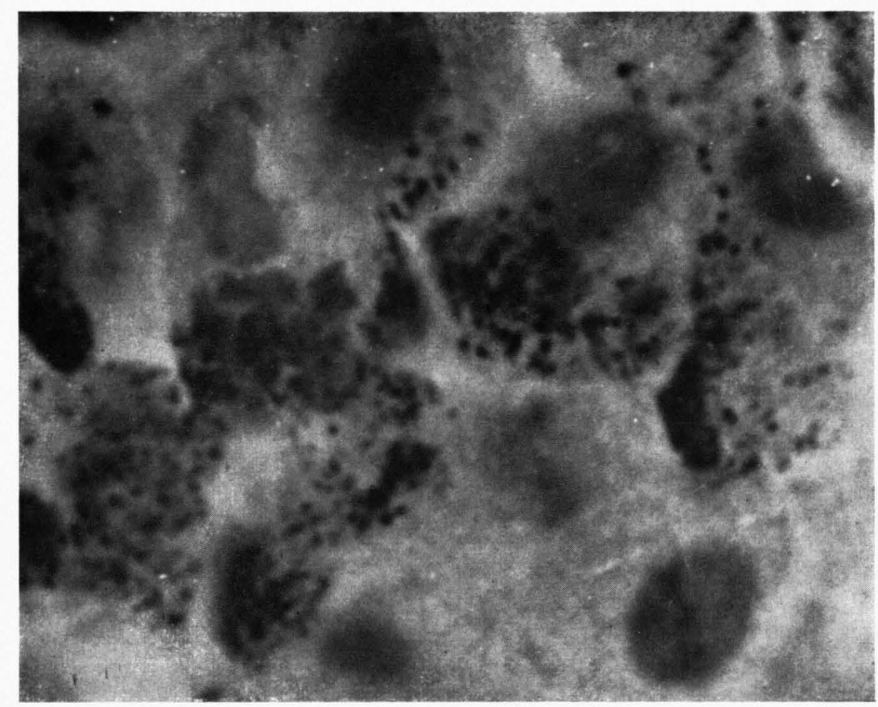

Abb. 3. Pankreas von nativ gefärbtem Normaltier, Inselausschnitt, Gefrierschnitt, Schnittdicke $6 \mu$, kurz überfärbt mit wässerigem Hämatoxylin, Vergrößerung $810 \times$, Immersion, Grünfilter. Auf dünneren Schnitten lassen sich bei stärkerer Vergrößerung die Granula sehr schön darstellen. Die mit Hämatoxylin angefärbten Zellkerne erlauben einen Größenvergleich. 


\begin{tabular}{|c|c|c|c|c|c|}
\hline $\begin{array}{l}\text { Tier } \\
\text { Nr. }\end{array}$ & $\begin{array}{c}\text { Gewicht } \\
\mathrm{kg}\end{array}$ & $\begin{array}{l}\mathrm{Zn} \\
\gamma / \mathrm{g}\end{array}$ & $\begin{array}{c}\mathrm{N}_{2} \\
\mathrm{mg} / \mathrm{g}\end{array}$ & Histochemischer Befund & Bemerkung \\
\hline 300 & 2,8 & $\begin{array}{l}23,5 \\
29,3\end{array}$ & - & - & \\
\hline 301 & 2,0 & $\begin{array}{l}25,7 \\
26,3\end{array}$ & - & ++++ & \\
\hline 302 & 2,8 & $\begin{array}{l}25,3 \\
32,0\end{array}$ & - & +++-++++ & \\
\hline 303 & 2,4 & $\begin{array}{l}35,0 \\
44,6\end{array}$ & - & +++ & \\
\hline 304 & 2,2 & $\begin{array}{l}91,0 \\
88,0\end{array}$ & - & +++-++++ & fettes Tier \\
\hline 325 & - & $\begin{array}{l}26,5 \\
43,3\end{array}$ & 41,1 & +++-++++ & \\
\hline 326 & - & $\begin{array}{l}50,5 \\
41,3\end{array}$ & 39,4 & ++++ & \\
\hline 343 & - & 19,3 & 27,8 & +++ & $\begin{array}{l}\text { Tuberkulom } \\
\text { am Bauch }\end{array}$ \\
\hline 344 & - & $\begin{array}{l}29,2 \\
33,3\end{array}$ & 21,4 & +++-+++ & \\
\hline 345 & - & $\begin{array}{l}32,6 \\
22,0\end{array}$ & 32,0 & ++ & \\
\hline 346 & - & $\begin{array}{l}30,7 \\
35,3\end{array}$ & 32,8 & +++ & \\
\hline 348 & 1,8 & 40,2 & 28,6 & ++++ & \\
\hline 349 & 1,9 & $\begin{array}{l}42,9 \\
48,2\end{array}$ & 32,3 & ++++ & $\begin{array}{c}\text { Pankreas diffus } \\
\text { mit Fett durchsetzt }\end{array}$ \\
\hline 351 & 2,2 & $\begin{array}{l}35,1 \\
26,4\end{array}$ & 35,0 & ++++ & \\
\hline 352 & 2,2 & $\begin{array}{c}37,7 \\
34,1 \\
\\
m=33,4 \\
\pm 24,9 \gamma / g\end{array}$ & $\begin{array}{c}33,1 \\
\\
m=32,4 \\
\pm 16,2 \mathrm{mg} / \mathrm{g}\end{array}$ & ++-+++ & \\
\hline
\end{tabular}

Tab. 2. 24-48 Stdn. hungernde Kaninchen.

der mittleren Abweichung vom Mittelwert des Gesamtpankreaszinks liegen.

Die mittleren Abweichungen $(=3 \sigma)$ von den Mittelwerten des Gesamtpankreaszinks betragen bei den drei Versuchsgruppen zwischen $\pm 24,3$ und $28,8 \gamma / \mathrm{g}$ oder bei einer gemeinsamen Mittelwertsbildung aller drei Gruppen: $m=38,8 \pm 29,7 \gamma /$ g. Die in Abb. 4 graphisch nach Klassen angegebenen Werte der verschiedenen Versuchsgruppen zeigen eine ziemlich gleichmäßige Verteilung; es läßt sich leicht errechnen, daß auch bei einer wesentlichen Erhöhung des Materials kaum ein statistisch signifikanter Unterschied zu erwarten ist.

Der Stickstoff wurde in den meisten Fällen gleichzeitig bestimmt, um eine Veränderung im Flüssig- keitsgehalt der Gewebe, z. B. bei den mit reichlicher Flüssigkeitszufuhr verbundenen Glucosegaben, auszuschließen. Es waren aber keine signifikanten Differenzen zu beobachten.

Das vorliegende Material erlaubt keine weiteren Schlüsse, z. B. zwischen dem Zinkgehalt des Pankreas und Alter, Geschlecht oder Gewicht der Versuchstiere.

C) Das Verhalten des Blutzuckers bei Kaninchen im Verlauf der Glucosebelastung

Die Blutzuckerwerte bewegten sich im allgemeinen um $200 \mathrm{mg} \%$. Bei einigen Tieren zeigte der Blutzucker gegen Ende der 10 Stdn. dauernden Belastung 


\begin{tabular}{|c|c|c|c|c|c|}
\hline $\begin{array}{l}\text { Tier } \\
\text { Nr. }\end{array}$ & $\begin{array}{c}\text { Gewicht } \\
\mathrm{kg}\end{array}$ & $\begin{array}{l}\mathrm{Zn} \\
/ / \mathrm{g}\end{array}$ & $\begin{array}{c}\mathrm{N}_{2} \\
\mathrm{mg} / \mathrm{g}\end{array}$ & Histochemischer Befund & Bemerkung \\
\hline 310 & 2,9 & $\begin{array}{l}14,7 \\
63,3\end{array}$ & 24,6 & ++-+++ & gravide, fett \\
\hline 311 & 2,9 & $\begin{array}{l}42,0 \\
48,0\end{array}$ & 38,9 & +-++ & \\
\hline 312 & 1,9 & $\begin{array}{l}45,3 \\
47,9\end{array}$ & 32,6 & +-++ & \\
\hline 313 & 1,9 & $\begin{array}{l}46,3 \\
46,0\end{array}$ & 36,8 & ++ & \\
\hline 314 & 1,9 & $\begin{array}{l}49,8 \\
51,3\end{array}$ & 29,7 & ++ & \\
\hline 315 & - & $\begin{array}{l}26,1 \\
31,5\end{array}$ & 31,4 & + & \\
\hline 316 & 2,6 & $\begin{array}{l}26,1 \\
26,7\end{array}$ & 30,9 & + & \\
\hline 317 & 2,3 & $\begin{array}{l}32,8 \\
31,4\end{array}$ & 34,5 & +-++ & \\
\hline 318 & 2,0 & $\begin{array}{l}21,5 \\
29,0\end{array}$ & 30,3 & ++ & \\
\hline 319 & 2,1 & $\begin{array}{l}39,3 \\
41,8\end{array}$ & 29,2 & +-++ & \\
\hline 353 & 1,9 & $\begin{array}{l}50,3 \\
51,2\end{array}$ & 32,5 & +-++ & \\
\hline 354 & 1,5 & $\begin{array}{l}41,2 \\
48,1\end{array}$ & 29,0 & + & $\begin{array}{l}\text { Pankreas } \\
\text { gelblich, fettreich }\end{array}$ \\
\hline 355 & 1,8 & $\begin{array}{l}27,5 \\
32,1\end{array}$ & 35,5 & +-++ & \\
\hline 356 & 1,7 & $\begin{array}{l}33,4 \\
39,1\end{array}$ & 31,3 & $0-+$ & \\
\hline 357 & 2,3 & $\begin{array}{l}56,5 \\
56,9\end{array}$ & 28,0 & $0-+$ & $\begin{array}{c}\text { Pankreas } \\
\text { gut abgesetzt }\end{array}$ \\
\hline 358 & 3,3 & $\begin{array}{l}42,0 \\
40,6\end{array}$ & 30,3 & ++ & $\begin{array}{c}\text { Pankreas } \\
\text { diffus verfettet }\end{array}$ \\
\hline 359 & 3,1 & $\begin{array}{l}40,2 \\
35,6\end{array}$ & 28,4 & +-++ & $\begin{array}{c}\text { Pankreas } \\
\text { diffus verfettet }\end{array}$ \\
\hline 360 & 2,6 & $\begin{array}{l}29,7 \\
17,0\end{array}$ & 22,0 & $0-+$ & $\begin{array}{c}\text { Pankreas } \\
\text { diffus verfettet }\end{array}$ \\
\hline \multirow[t]{2}{*}{361} & 2,9 & $\begin{array}{l}40,6 \\
50,1\end{array}$ & & + & $\begin{array}{c}\text { gravide, Pankreas } \\
\text { gut abgesetzt }\end{array}$ \\
\hline & & $\begin{array}{l}m=39,3 \\
\pm 28,8 \gamma / \mathrm{g}\end{array}$ & $\begin{array}{c}m=30,9 \\
\pm 11,7 \mathrm{mg} / \mathrm{g}\end{array}$ & & \\
\hline
\end{tabular}

Tab. 3. Glucosebelastete Tiere.

eine Tendenz zum Ansteigen, z. Tl. bis auf Werte von $400-500 \mathrm{mg} \%$. Dieser Befund wurde von uns als Ausdruck einer zunehmenden Erschöpfung des Inselapparates gewertet. Es wurden bei überlebenden Kontrollen aber keine irreversiblen Schäden des Inselapparates gefunden; solche waren allerdings bei der verhältnismäßig kurzen Glucosebelastung auch nicht zu erwarten.

An dieser Stelle sei auf das atypische Verhalten des
Blutzuckers nach Alloxangaben bei in gleicher Weise mit Glucose vorbehandelten Tieren erinnert. Derartige Tiere zeigten kaum eine Hypoglykämie, wie sie sonst nach Alloxangaben häufig zum Tode der Versuchstiere führt ${ }^{2}$. Das Fehlen der Hypoglykämie wurde von uns damit erklärt, daß dem Inselapparat durch die vorhergehende Glucosebelastung das Insulin weitgehend entzogen worden war, während bei normalen unvorbehandelten Tieren durch das in- 
jizierte Alloxan die Insulinvorräte aus den zerstörten B-Zellen an das Blut abgegeben werden.

\section{Diskussion}

Es wurde gezeigt, daß in vivo bei Kaninchen sehr enge Beziehungen zwischen Insulin und Zink bestehen. Die Granula der Inselzellen, die als morphologisches Äquivalent für das Insulin angenommen werden müssen, enthalten sehr viel Zink. Außerhalb der Granula ist in den Inselzellen beim Kaninchen kein Zink histochemisch nachzuweisen. Der quantitativ bestimmte Gesamtzinkgehalt des Pankreas wird aber entgegen der Annahme älterer Autoren durch die starke lokale Anreicherung innerhalb der Inselzellen nicht wesentlich beeinflußt. Es muß allerdings hierzu bemerkt werden, daß sicher nicht das gesamte nach der Mineralisation quantitativ bestimmbare Zink histochemisch nachweisbar ist. Vi k b la d h ${ }^{18}$ konnte z. B. nur einen Teil des Serumzinks mit Dithizon extrahieren. Eine derartige Extraktion setzt genau wie die histochemische Darstellung des Gewebezinks mit Diphenyl-thiocarbazon oder Diphenyl-thiocarbazid eine Zinkkomplexbildung voraus. Zinkhaltige Insulinkristalle färbten sich jedoch nach unserer Beobachtung gleich stark an wie die Inselzellgranula.

Bei funktionsmäßig extremen Zuständen der Langerhansschen Inseln verhält sich das dort histochemisch nachweisbare Zink entsprechend dem Insulingehalt. Nach sehr sorgfältigen Untersuchungen von $\mathrm{Best}, \mathrm{Campbell}, \mathrm{Ha}$ is t und $\mathrm{Ham}$ besteht eine direkte Abhängigkeit zwischen der Dichte und der Quantität der B-Zellen-Granula und dem Insulingehalt des Pankreas ${ }^{19}$. Bei vermehrtem Insulinbedarf werden die B-Zellen mehr und mehr degranuliert. Unter extremen experimentellen Bedingungen können allein durch intravenöse Glucosegaben Inselzellnekrosen erzeugt werden 20,21,22. Dementsprechend fanden wir am meisten zinkhaltige Granula bei hungernden bzw. bei normal gefütterten Tieren. Dabei waren die verschiedenen Inseln desselben Pankreas nicht immer gleich zinkreich. Auch diese Beobachtung entspricht älteren Feststellungen über das Verhalten der Granula und wird auf eine wechselnde Inanspruchnahme der Inseln, auf deren alternierende Funktion zurückgeführt. Die Beobachtung von Ferner über das Vorhandensein von sogenannten Sperrwülsten am Abgang der Läppchenarterien recht

18 I. V i k b l a d h, Suppl. II, Scand. J. Clin. Lab. Invest. [1951].

19 C. H. B est, J. Campbell, R. E. Ha is t u. A. W. Ha m, J. Physiology 101, 17 [1942]. fertigt diese Annahme. Nach intensiven, kombiniert peroralen und intravenösen Glucoseblastungen fanden wir analog zu dem bereits angeführten Verhalten der Granula eine Abnahme des Zinks in den Inseln. Das histochemisch nachweisbare Zink und das Insulin nehmen bei der Beanspruchung der Inseln also gemeinsam ab. - Nach der Injektion diabetogener Substanzen verhält sich das Zink ebenfalls parallel dem Insulingehalt der Inseln. Es nimmt deutlich im Verlauf der anfänglichen Hyperglykämie ab, und bei ausgebildetem Diabetes enthalten die Inseln kaum mehr Zink. Für die letztere Beobachtung wären zwei Erklärungen denkbar: einmal könnte der hohe diabetische Blutzuckergehalt die physiologische Anreicherung von Zink in den Langerhansschen Inseln verhindern, oder aber der Mangel von funktionsfähigem Inselgewebe ist die Ursache der fehlenden Zinkspeicherung.

Welche Bedeutung mag nun dem Zink im Insulin oder dessen unmittelbarer Vorstufe in den Langerhansschen Inseln zukommen? Nach älteren Untersuchungen wirkt auch praktisch zinkfreies Insulin genau so blutzuckersenkend wie zinkhaltiges. Zink bewirkt anscheinend unter bestimmten Voraussetzungen eine protrahierte Resorption von injiziertem Insulin. Es ist außerdem sehr unwahrscheinlich, daß die große Menge des histochemisch nachweisbaren Inselzinks eine Bedeutung für die biologische Insulinsynthese besitzt. Nach dem oben beschriebenen Verhalten liegt es am nächsten, eine Funktion des Zinks bei der basalen Insulinregulation anzunehmen.

Nach Untersuchungen von $\mathrm{Co} \mathrm{h} \mathrm{n}$ ist bekannt, daß Proteine und speziell Insulin durch Zink bei physiologischem $p_{\mathrm{H}}$ gefällt werden: mit diesem Effekt konkurrieren bestimmte Anionen, nach $\mathrm{H}$ a ll as - M ölle r z.B. Phosphat- und Citrationen. Nun liegt ja das physiologische Insulindepot in den Inselzellen in einer engen topographischen Beziehung zum histochemisch nachweisbaren Zink ungelöst in Form der sogenannten Granula vor. Die Voraussetzung für eine Abgabe des Hormons an das Blut wäre dessen Überführung in eine lösliche Form und vorher eine Änderung der Zinkbindung. Es ist anzunehmen, daß die große Oberflächenaktivität derartiger Granula bei der Zinkbindung bzw. deren Änderung nicht ohne Bedeutung ist.

Nachdem frühere Untersuchungen gezeigt haben,

20 E. Anderson u. J. A. Long, Endocrinology 40, 92 [1947].

${ }_{21}$ S. St. B a rron u. D. S t a t e, Arch. Pathology 48, 297 [1949].

22 F. D. W. Lukens, Z. klin. Med. 145, 201 [1949]. 
daß die Glucose anscheinend der spezifische Reiz für die Insulinabgabe ist, muß man schließen, daß die Erhöhung der Blutglucose direkt oder wahrscheinlicher indirekt die Ursache für die Änderung der Zinkbindung des Insulins ist. Nach $\mathrm{He} f \mathrm{fte} r$ hat Zink eine spezifisch chemische Affinität zu verschiedenen biologischen Schwermetallkomplexbildnern, zu Aminosäuren, Zuckerphosphatsäuren, Lipoiden, Brenzcatechinen, deren Derivaten, SH-haltigen Substanzen und zu Derivaten des Blutfarbstoffs ${ }^{23}$. Ähnliche Substanzen kommen als konkurrierende Komplexbildner mit dem Insulin-Zink in Frage. Vielleicht deutet die Beobachtung von $\mathrm{S}$ ti e r auf die intrainsuläre Anreicherung derartiger Substanzen; er fand, daß die Langerhansschen Inseln von normalen Tieren Triphenyltetrazoliumchlorid kaum reduzieren, während die Inseln mit Glucose belasteter Tiere eine wesentlich stärkere Formazanbildung zeigen ${ }^{24}$.

In gewisser Weise analog zu den Beobachtungen bei der Entstehung des Dithizon- und Alloxandiabetes lassen sich die Vorgänge bei der basalen Insulinregulation durch die Blutglucose bisher wie folgt formu-

23 Hdbch. exp. Pharmakol., 3. Bd., 3. Tl., S. 1909 [1934].

24 A. S ti e r, Z. Anatomie Entwicklungsgesch. 116, 399 [1952]. lieren: Das Insulin liegt auf Grund seiner Zinkbindung in unlöslicher Form in den Inseln vor. Nach der Erhöhung des Blutglucosespiegels kommt es an der Oberfläche der Granula zu einer konkurrierenden Komplexbildung zwischen dem Insulinzink einerseits und Substanzen, die infolge der Vermehrung der Blutglucose auftreten, andererseits. Dadurch wird das Insulin in eine lösliche Form übergeführt und somit die Voraussetzung für dessen Abgabe an das Blut gegeben.

Bei der ausschließlichen Betrachtung der sogenannten basalen Insulinregulation wurden in der vorliegenden Arbeit bewußt weitere Faktoren außer acht gelassen, die im Gesamtorganismus sicher diese Vorgänge zusätzlich beeinflussen, wie z. B. zentral-nervöse und weitere hormonale Einflüsse.

Diese Untersuchungen erscheinen auch von Interesse für die Klinik des Diabetes, da Störungen der Insulinregulation leicht zu einem Mangel an Insulinreserven oder direkt zu dem Bild eines Insulinmangelzustandes führen können.

Die Untersuchungen wurden durch die Hilfe der Deutschen Forschungsgemeinschaft ermöglicht.

\section{NOTIZEN}

Zur Kenntnis von Siliciumditellurid (II. Mitt. über Siliciumchalkogenide) ${ }^{1}$

Von Alarich Weis und Armin Weis s

Eduard-Zintl-Institut für anorganische und physikalische Chemie der Technischen Hochschule Darmstadt

(Z. Naturforschg. 8 b, 104 [1953]; eingeg. am 26. Januar 1953)

Siliciumditellurid, $\mathrm{SiTe}_{2}$, wurde durch Erhitzen von Silicium und Tellur auf $1050-1070^{\circ} \mathrm{C}$ im Hochvakuum dargestellt. Die Verbindung bildet rote Plättchen, die sich an der Luft unter Bildung von $\mathrm{SiO}_{2}$ und $\mathrm{H}_{2}$ Te zersetzen. Bei der Zersetzung im Hochvakuum bei etwa $1200^{\circ} \mathrm{C}$ entsteht neben Silicium und Tellur grauweißes, kubisches Siliciummonotellurid SiTe.

Die Analyse ergab im Mittel 10,0\% Si (theor. 9,91\%) und $90,0 \% \mathrm{Te}$ (theor. 90,09\%). Die Einwaagen zu den Analysen und die Röntgenuntersuchungen wurden unter Luftausschluß ausgeführt. Die Dichte ergab sich zu 4,39.

$\mathrm{SiTe}_{2}$ kristallisiert im Cadmiumjodidtyp mit den Achsen $a=4,28 \pm 0,01 \AA$ und $c=6,71 \pm 0,01 \AA$. Die Intensitäten der Interferenzen auf den Drehkristall-Weissenberg- und Pulveraufnahmen ergaben einen $z$-Parameter von 0,265

1 A. W e is s u. A. W e is s, Z. Naturforschg. 7 b, 483 [1952]. $\pm 0,005$. Daraus folgt ein kürzester Si-Te-Abstand von $3,04 \AA$. Dieser Abstand ist größer als die Summe der Ionenradien nach Gold s chmidt mit $2,50 \AA$ und entspricht eher dem Abstand für metallische Bindung.

Bei der Darstellung des $\mathrm{SiTe}_{2}$ wurden gelegentlich grauweiße Fasern erhalten, welche eine faserige Modifikation des SiTe, darstellen; diese ist wahrscheinlich mit dem faserigen $\mathrm{SiSe}_{2}{ }^{1}$ isotyp.

Eine ausführliche Veröffentlichung erscheint in Kürze in der Zeitschrift für anorganische Chemie.

\section{Zur Kenntnis von Siliciumdiselenid-Glas (III. Mitt. über Siliciumchalkogenide) ${ }^{1}$}

Von Alarich Weis und Armin Weis s

Eduard-Zintl-Institut für anorganische und physikalische Chemie der Technischen Hochschule Darmstadt

(Z. Naturforschg. 8 b, 104-105 [1953]; eingeg. am 26. Januar 1953)

Glasiges Siliciumdiselenid, $\mathrm{SiSe}_{2}$, bildet sich, wenn man kristallisiertes, faseriges $\mathrm{SiSe}_{2}{ }_{2}$ im evakuierten Quarz-

1 2. Mitt.: A. W e i s s u. A. W e is s, Z. Naturforschg. 8 b, 104 [1953], voranstehend.

2 A. W e is s u. A. We is s, Z. Naturforschg. 7 b, 483 [1952]. 